\title{
THE ORDER TOPOLOGY FOR FUNCTION LATTICES AND REALCOMPACTNESS
}

\author{
W.A. FELDMAN \\ Department of Mathematics; University of \\ Arkansas, Fayetteville, AR 72701 \\ and \\ J.F. PORTER \\ Department of Mathematics, University of \\ Arkansas, Fayetteville, AR 72701 \\ (Received June 12, 1980)
}

ABSTRACT. A lattice $\mathrm{K}(\mathrm{X}, \mathrm{Y})$ of continuous functions on space $\mathrm{X}$ is associated to each compactification $\mathrm{Y}$ of $\mathrm{X}$. It is shown for $\mathrm{K}(\mathrm{X}, \mathrm{Y})$ that the order topology is the topology of compact convergence on $X$ if and only if $X$ is realcompact in $Y$. This result is used to provide a representation of a class of vector lattices with the order topology as lattices of continuous functions with the topology of compact convergence. This class includes every $C(X)$ and all countably universally complete function lattices with 1 . It is shown that a choice of $K(X, Y)$ endowed with a natural convergence structure serves as the convergence space completion of $\mathrm{V}$ with the relative uniform convergence.

KEY WORDS AND PHRASES. Order topology, relative uniform convergence, realcompactness, universally complete function lattice, convergence space completion. 2980 MATHEMATICS SUBJECT CLASSIFICATION CODES. 54A20, 54C40, $46 A 40$

\section{INTRODUCTION.}

In this paper we will study a broad class of real function lattices which we call "2-universally complete." For this class we will show that the order topology $T_{0}$ (also called the order bound topology and the relative uniform topology) 1s the topology of compact convergence in an appropriate representation (Theorem2). We will show (Proposition 3) that the 2-universally complete lattices include the 
lattices $C(X)$, all continuous real-valued functions on $X$, and all countably universally complete lattices containing 1. (An example of this latter type is discussed in Example 1.)

The proof of Theorem 2 requires a construction which is studied independently in $\S 1$. In particular, sublattices $K(X, Y)$ of $C(X)$ for compactifications $Y$ of $X$ are investigated. Theorem 1 states that the order topology for $K(X, Y)$ Is the topology of compact convergence on $X$ if and only if $X$ is realcompact In Y. (This concept of realcompactness was studied in [10].)

Since the order topology is the finest locally convex topology in which every relatively uniformly convergent net converges (see [5]), in 53 we consider the 2-universally complete function lattice $V$ with relative uniform convergence as a convergence function lattice $\nabla_{\rho}$, without reference to its assocfated order topology. We show (Theorem 4) that $K(X, Y)$ endowed with a natural convergence structure serves as the completion in the convergence space sense of $v_{\rho}$.

We remark that (assuming without loss of generality that $X$ is realcompact) It can be seen directly that the order topology is the topology of compact convergence for the lattice $C(X)$. This follows from [13, p. 124] since every positive linear functional is continuous with respect to the topology of compact convergence (see [6]) and since $C(X)$ with the topology of compact convergence is barrelled (see [11]).

2. THE ORDER TOPOLOGY FOR $R(X, Y)$

Let $Y$ be a compact Hausdorff space and $X$ a dense subspace. We denote by $F(X, Y)$ the set of all nonnegative extended real-valued continuous functions on $Y$ which are finite on $X$. For $f$ in $F(X, Y)$ we let $\Lambda_{f}$ be the set in $Y \backslash X$ where $f$ is infinite. We set

$$
K(X, Y)=U\left\{C\left(Y \backslash \Lambda_{f}\right): f \in F(X, Y)\right\} \text {. }
$$

Since $X$ is dense in each $Y \backslash \Lambda_{f}$, by restricting the functions in $K(X, Y)$ to $X$ we can view $K(X, Y)$ as a sublattice (and also a subalgebra) of $C(X)$. 
LEMMA 1. For each function $g$ in $K(X, Y)$ there is a function $f$ in $F(X, Y)$ such that $8 \leq f$.

PROOF. Given $g$ in $K(X, Y)$ there is a function $h \geq 0$ in $F(X, Y)$ such that $g$ is in $C\left(Y-\Lambda_{h}\right)$. We consider the compact subsets

$$
A_{n}=h^{-1}[n-1, n]
$$

and $\quad B_{n}=h^{-1}[0, n-2] \cup h^{-1}[n+1, \infty]$

of $Y(n=1,2, \ldots)$ with the understanding that $B_{1}=h^{-1}[2, \infty]$. Using separating functions on $Y$, one can construct for each $n$ a continuous function $f_{n}$ such that

$$
f_{n}(x)=\sup \left\{g(z): z \in A_{n}\right\} \text { for } x \text { in } A_{n}
$$

and

$$
f_{n}(x)=h(x) \text { for } x \text { in } B_{n} \text {. }
$$

On $\mathrm{Y}-\Lambda_{\mathrm{h}}$ the function $\mathrm{f}$ defined by

$$
f(x)=\sup \left\{f_{n}(x): n=1,2, \ldots\right\}
$$

is continuous, since at each point $x$ in $Y-\Lambda_{h}$ there is a neighborhood of $x$ on which $f$ is the supremum of finitely many functions $f_{n}$. Moreover, $f \geq h$ on $Y \backslash \Lambda_{h}$ and hence extends continuously to $Y\left(1 . e ., f(x)=\infty\right.$ for $x$ in $\left.\Lambda_{h}\right)$. Thus $f$ is in $F(X, Y)$, and $g \leq f$.

For $Y$ a compact Hausdorff space and $X$ a dense subspace of $Y$, we will say that $X$ is realcompact in $Y$ if

$$
\mathrm{X}=\bigcap\left\{\mathrm{Y}-\Lambda_{\mathrm{f}}: \text { f } \in \mathrm{F}(\mathrm{X}, \mathrm{Y})\right\}
$$

This concept has been considered by Lorch in $[10]$. Where $B X$ denotes the Stone-Cech compactification of $x$, we note that $X$ is realcompact if and only if $X$ is realcompact in $B X$. If $X$ is realcompact in $Y$ it follows that $X$ is realcompact, since $Y$ is a quotient of $B X$. On the other hand, the real line $X$ with its discrete topology is realcompact but not realcompact in its one-point compactification $Y$ : The set $\Lambda_{f}$ is empty for each $f$ in $F(X, Y)$ since $X$ is not $\sigma$ - compact, implying

$$
\bigcap\left\{\mathrm{Y}>\Lambda_{\mathrm{f}}: \mathrm{f} \in \mathrm{F}(\mathrm{X}, \mathrm{Y})\right\}=\mathrm{Y} \text {. }
$$

We note that the following proposition is also a consequence of work done in [8]. 
PROPOSITION 1. A completely regular space $X$ is realcompact in each of its compactifications if and only if it is Lindelöf.

PROOF. Suppose $X$ is Lindelöf, $Y$ is a compactification of $X$ and $P \in Y-X$. Arguing as in [9], for each $x$ in $X$ we define a Urysolin function $h_{X}$ on $Y$ such that $h_{X}(x)=1, h_{x}(p)=0$ and $0 \leq h_{x} \leq 1$. Then $\left\{h_{x}^{-1}\left(\frac{1}{2}, \infty\right)\right.$ : $X \in X\}$ is an open cover of $X$ having a countable subcover corresponding to functions $\left\{h_{n}\right\}_{n=1}^{\infty}$. Let $h=\left[h_{n} / 2^{n}\right.$, a non-negative member of $c(Y)$ which is strictly positive on $X$ and zero at $p$. Thus $p$ is in $\Lambda_{1 / h}$, showing that $X$ is realcompact in $Y$. Conversely, if $X$ is not Lindelöf, by [9] there is a compact set $K$ in $B X-X$ which is not contained in a zero set in $B X-X$. Let $Y$ be that quotient of $B X$ obtained by identifying the points of $K$. Since the Image of $K$ in $Y$ cannot be contained in a zero set in $Y \backslash X, X$ is not realcompact in $Y$.

The subscript co will denote the topology of compact convergence and the subscript $T_{0}$ will denote the order topology. For a completely regular space $x$ with realcompactification $\nu \mathrm{X}$, as noted in the introduction, $\mathrm{C}_{\mathrm{T}_{0}}(\nu \mathrm{X})=\mathrm{C}_{\mathrm{co}}(\nu \mathrm{X})$. since $C_{c o}(X) \neq C_{c o}(v X)$ if $X$ is not realcompact, we conclude that $C_{T_{0}}(x)=C_{c o}(x)$

1f and only if $\dot{X}$ is realcompact (In $B X$ ). We provide the following generalization, noting that $K(X, B X)$ is $C(X)$.

THEOREM 1. Let $Y$ be a compact Hausdorff space and $X$ be a dense subspace of $Y$. Then in $K(X, Y)$ the order topology coincides with the topology of compact convergence on $X$ if and only if $X$ is realcompact in $Y$.

PROOF. Setting

$$
Z=\cap\left\{Y-\Lambda_{f}: \quad f \in F(X, Y)\right\},
$$

we note that

$$
K(X, Y)=K(Z, Y) \text {. }
$$

We abbrevlate $K(X, Y)$ and $F(X, Y)$ to $K$ and $F$. The subscript $\rho$ will denote the relative unfform convergence structure. To complete the proof, we show that 
the topology of $\mathrm{K}_{\mathrm{T}}$ is the topology of compact convergence on $\mathrm{z}$. Let $\left\{\mathrm{f}_{\alpha}\right\}$ be a net convergent to zero in $\mathrm{K}_{\rho}$. (As noted in the Introduction, $T_{0}$ is the finest locally convex topology in which every net convergent in $K_{\rho}$ converges.) There is a $g \geq 0$ in $K$ such that for all $n$,

$$
\left|f_{\alpha}\right| \leq \frac{1}{n} \cdot g \quad\left(\alpha \geq \alpha_{n}\right) .
$$

Clearly $\left\{\frac{1}{n} \cdot g\right\}$ converges to zero in $C_{c o}\left(Y-\Lambda_{g}\right)$ and hence in $C_{c o}(Z)$. Since $C_{c o}(Z)$ is a topological vector lattice, $\left\{_{\alpha}\right\}$ converges in $C_{c o}(Z)$. Thus the map from $\mathrm{K}_{\mathrm{T}_{0}}$ into $\mathrm{C}_{\mathrm{co}}(\mathrm{Z})$ is continuous. To show that $\mathrm{T}_{0}$ is coarser than the topology of compact convergence on $Z$, let $U$ be a closed, absolutely convex, solid neighborhood of zero in $K_{T_{0}}$. We remark that for $f \in F$, the inclusion map from $C_{c o}\left(Y \backslash \Lambda_{f}\right)$ into $K_{T_{0}}$ is continuous. This follows from the fact that the map from $C_{\rho}\left(Y-\Lambda_{f}\right)$ and hence from $C_{T_{0}}\left(Y>\Lambda_{f}\right)$ into $K_{T_{0}}$ is continuous. (As noted in the introduction, $\mathrm{C}_{\mathrm{T}_{\mathrm{o}}}\left(\mathrm{Y} \backslash \Lambda_{\mathrm{f}}\right)$ is $\left.\mathrm{C}_{\mathrm{co}}\left(\mathrm{Y} \backslash \Lambda_{\mathrm{f}}\right)\right)$ Since $C_{C O}(Y)$ is $C_{c o}\left(Y-\Lambda_{1}\right)$ and $U$ is solid,

$$
\mathrm{U} \supseteq\left\{\mathrm{g} \in \mathrm{K}:|| \mathrm{g}||_{\mathrm{z}} \leq \delta\right\}
$$

where $\|\cdot\|_{Z}$ denotes the supremum on $z$, and $\delta$ is a fixed positive number. The following argument uses techniques found in [11]. We will call a compact set $G$ in $Y$ a support set for $U$ if for $f$ in $F, f$ is in $U$ whenever its restriction to $G$ vanishes. For example, $Y$ is a supportset for $U$ and, assuming $U$ does not contain $F$ (if $U \geq F$ then $U=K$ by Lemma 1), the empty set is not a support set for $U$. We note several properties of support sets for $U$ which will be needed.

(a) Let $G$ be a support set for $U$. If $f$ is in $F$ with

$$
\|f\|_{G}<\delta / 2 \text { then } f \text { is in } U \text {. }
$$

To see this, consider the function $g=(f-\delta / 2)$ Vo. Although $F$ is not a vector lattice, $g$ is clearly in $F$. Since || $2 g \|_{G}=0,2 g$ is in $U$; since $\|2(f-g)\|_{Z} \leq \delta, 2(f-g)$ is in $U$. Thus by the convexity of $U, f$ is in $U$. 
(b) Let $G$ be a compact subset of $Y$. If for $h$ in $F, h$ is in $U$ whenever $h$ vanishes on a neighborhood of G, then $G$ is a support set for $U$. To see this, suppose $f \in F$ vanishes on such a set $G$. The function $\mathrm{g}=$ $(f-\delta / 2)$ v0 vanishes on $f^{-1}(-\delta / 2, \delta / 2)$, a neighborhood of G. Thus $2 g$ is in $U$. Since again $2(f-g)$ is in $U$ we obtain $f \varepsilon U$.

(c) The intersection of two support sets for $U$ is a support set for $U$.

To see this, let $G$ and $H$ be support sets for $U$ and let $f$ in $F$ vanish on a neighborhood $\mathrm{W}$ of $\mathrm{G} \cap \mathrm{H}$. If $f$ is bounded there is a $g$ in $\mathrm{C}(\mathrm{Y}) \cap \mathrm{F}$ such that $\|g\|_{G}=0$ and $g(x)>f(x)$ for $x$ in $H-W$. Since $\|g \wedge f\|_{G}=0,2(g \wedge f)$ is in $U$, and since $\|(f-g) \vee 00\|_{H}=0,2[(f-g) \vee 0]$ is in $U$. Thus by the convexity of $U$, $f$ is in $U$. Now suppose $f$ is not bounded. Then $f$ is the limit in $K_{T_{0}}$ of the bounded functions $\{f \wedge n 1\}$ since this sequence converges to $f$ in $C_{c o}\left(Y \backslash \Lambda_{f}\right)$. Each $f \wedge_{n} 1$ is in $U$ and $U$ is closed, so that $f$ is in $U$.

(d) The intersection $\mathrm{S}$ of all support sets for $U$ is a support set for $U$.

To see this, let $f$ in $F$ vanish on a neighborhood $W$ of $S$. Since $Y-W$ is compact it is covered by the complements of finitely many support sets for $U$. Thus $f$ vanishes on the intersection of these finitely many support sets and is in $U$ by (c).

We prove that the intersection $S$ of all support sets for $U$ is contained in $Z$. Let $p$ be in $Y \backslash Z$. Then $h(p)=+\infty$ for some $h$ in $F$. Since the inclusion map from $\mathrm{C}_{\mathrm{co}}\left(\mathrm{Y}-\Lambda_{\mathrm{h}}\right)$ into $\mathrm{K}_{\mathrm{T}}$ is continuous, there is a compact subset $D$ of $Y \backslash \Lambda_{h}$ such that $U$ contains $\left\{g \in C\left(Y-\Lambda_{h}\right):|| g||_{D}<\varepsilon\right\}$ for some $\varepsilon>0$. Thus if $g$ is in $\mathrm{C}\left(\mathrm{Y}-\Lambda_{\mathrm{h}}\right) \cap_{\mathrm{F}}$ and vanishes on $\mathrm{D}$, then $\mathrm{g}$ is in $\mathrm{U}$. For any $f$ in $F$ which vanishes on $D$, since $f \wedge_{n l}$ is in $C\left(Y-\Lambda_{h}\right)$ we have $f \Lambda_{n} 1 \in U$. It follows from the fact that $U$ is closed that $f$ is in $U$. Thus $D$ is a support set for $U$ not containing $p$. We conclude that $S$ is contained

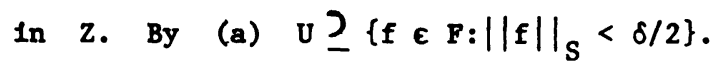


Let $g$ be any member of $k$ having $\|g\|_{S}<\delta / 2$. Then $g$ is in $C\left(Y-\Lambda_{h}\right)$ for some $h$ in $F$. By Lemma 1 we can assume $h \geq|g|$. There is a number $N$ large enough so that the closed set $h^{-1}[\mathrm{~N}, \infty)$ in $\mathrm{Y} \backslash \Lambda_{h}$ is disjoint from $S$. Since $Y \backslash \Lambda_{h}$ is normal there is a function $k$ in $C\left(Y \backslash \Lambda_{h}\right)$ which equals $|g|$ on $S$ and $h$ on $h^{-1}[N, \infty)$. Letting $f$ be $k v|g|$ on $Y \backslash \Lambda_{h}$ and $\infty$ on $\Lambda_{h}$, we conclude that $f$ is in $F$ since $f \geq h$ on $h^{-1}[N, \infty)$. Since $f=|g|$ on $S$, $f$ is in U. Since $f \geq|g|$ and $U$ is solid, $g$ is in $U$. Thus,

$$
\mathrm{U} \supseteq\left\{\mathrm{g} \in \mathrm{K}:\|\mathrm{g}\|_{\mathrm{S}}<\delta / 2\right\},
$$

a neighborhood of zero in the topology of compact convergence on $z$. This completes the proof.

\section{THE ORDER TOPOLOGY FOR A 2-UNIVERSALLY COMPLETE LATTICE}

We recall that an element $e$ in a vector lattice $V$ is said to be a weak order unit if for each $v$ in $v$

$$
\mathrm{v}=\mathrm{V}\{\mathrm{v} \wedge \mathrm{ne}: \mathrm{n}=1,2,3, \ldots\} \text {. }
$$

We will assume that $\mathrm{V}$ is a vector lattice having a weak order unit $e$ and that the real lattice homomorphisms on $V$ separate the points of $V$. We let $X$ be the set of lattice homomorphisms $x$ on $V$ such that $x(e)=1$ with the topology of pointwise convergence. We map $V$ into $C(X)$ by the usual Gelfand map $\hat{v}(x)=x(v)$ for all $x$ in $x$. We will refer to $x$ as the carrier space of v. The proof of the following proposition uses the techniques of Lemma 2 in [3]. PROPOSITION 2. The Gelfand mapping of $V$ into $C(X)$ is injective. PROOF. Suppose $\hat{v}=0$ for $v$ in $v$; thus, $x(v)=0$ for all $x$ in $x$. For each lattice homomorphism $\phi$ on $\mathrm{V}$ either $\phi(e)=0$ or $\phi / \phi(e)$ is in $\mathrm{X}$, so that

$$
\phi\left(v \wedge_{n e}\right)=\phi(v) \wedge_{n} \phi(e)=0 .
$$

Since the lattice homomorphisms separate $X, v^{\wedge}$ ne $=0$ for all $n$. Thus

$$
\left.v=V f_{v} \wedge \text { ne: } n=1,2, \ldots\right\}=0 \text {. }
$$

We will henceforth identify $V$ with its image in $C(X)$ and refer to it as a function lattice with 1 (the image of e). We also will not distinguish between functions in $C(X)$ and their extensions to functions from the 
Stone-Čech compactification $B X$ of $X$ to the extended real numbers.

We will have need for an additional condition. In a function lattice $V$

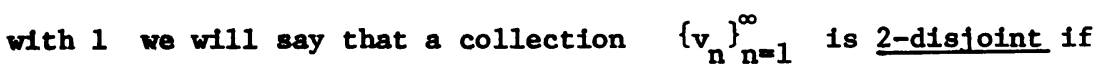

1. For each $n,\left|v_{n}\right| \wedge\left|v_{k}\right| \neq 0$ for at most two Indices $k$ distinct from $n$, and

2. for each $x$ in the carrier space $x$ of $v$ there is a $v_{n}$ such that $v_{n}(x) \neq 0$.

We will say that $V$ is 2-umiversally complete if each 2-disjoint collection has a supremum in $\nabla$.

For what follows we recall that a countably universally complete lattice 1s one in which the supremum exists for each collection $\left\{v_{n}\right\}_{n=1}^{\infty}$ satisfying $\left|v_{n}\right| \wedge\left|v_{j}\right|=0$ for $n \neq j$.

PROPOSITION 3. (a) The lattice $C(Y)$ for any completely regular space $Y$ is 2-universally complete. (b) Each countably universally complete function lattice with 1 is 2-universally complete.

PROOP. For (a), since the carrier space of $C(Y)$ is the realcompactification of $Y$ we may as well assume that $Y$ is realcompact. Given a 2-disfoint collection $\left\{f_{n}\right\}$ in $C(Y)$, at any point $y$ in $Y$ there is a function $f_{n}$ with $\left|f_{n}(x)\right|>0$ for all $x$ in a neighborhood $N$ of $y$. The pointwise supremum of the collection $\left\{f_{n}\right\}$ on $N$ thus involves at most three functions; one concludes that the pointwise supremum of $\left\{f_{n}\right\}$ is continuous, and thus the supremum of $\left\{f_{n}\right\}$ in $C(Y)$.

The proof of (b) follows from the observation that a 2-disjoint collection can be decomposed into three collections, each having a supremum by countable universal completeness.

We note that $C(R)$ ( $R$ the reals) is 2-universally complete but not countably universally complete: Letting $f_{n}$ be a continuous function which vanishes off $\left[\frac{1}{n+1}, \frac{1}{n}\right]$ and has value 1 at some point $x_{n}$, we obtain a collection $\left\{f_{n}\right\}_{n}=I$ satisfying $\left|f_{n}\right| \wedge\left|f_{j}\right|=0$ for $n \neq j$ whose supremum $f$ would clearly vanish on $(-\infty, 0)$ and yet $f(0)=\lim _{n \rightarrow \infty} f\left(x_{n}\right) \geq f_{n}\left(x_{n}\right)=1$. 
For a function lattice $V$ with 1 , we let $\widetilde{B X}$ denote the quotient space of the Stone-Čech compactification $\beta X$ of the carrier space $X$ which is induced by the equivalance relation $p \sim q$ if $v(p)=v(q)$ for all $v$ in $v$. The space $\widetilde{B X}$ is compact and (since $V$ separates $\widetilde{B X}$ ) Hausdorff. Note that $X$ is realcompact in $\widetilde{B X}$. Since $\mathrm{V}^{+}$is contained in $F(X, \widetilde{B X}), V$ is a sublattice of $K(X, \widetilde{B X})$. Of course, if $V$ separates $\beta X$ then $K(X, \widetilde{\beta X})=K(X, \beta X)=C(X)$.

We will provide an example of a 2-universally complete function lattice with 1 which is not uniformly dense in any function space $C(S)$. For other such examples see [7]. Furthermore, for the carrier space $X$ in this example, $\widetilde{\beta X} \neq \beta X$ and $K(X, \widetilde{\beta X})=\mathcal{L}$. For this purpose we will need the following lemma. We recall that a $\phi$-algebra is an archimedean lattice-ordered algebra over the reals with identity 1 which is a weak order unit.

L 2. Let $V$ be a $\phi$-algebra. Then every lattice homomorphism on $V$ is an algebra homomorphism.

PROOF. Let $\theta$ be a lattice homomorphism on $\mathrm{V}$. Then $\theta$ is a lattice homomorphism on the order ideal $I(1)$ generated by 1 , hence an extremal element in the continuous dual $I(1)^{\prime}$ of $I(1)$ in the order untt topology. For $g$ in $I(1), 0 \leq g \leq 1$, we define $\theta_{g}(f)=\theta(f g)$. Since for $f \geq 0$ in $I(1)$ we have $\theta_{g}(f) \leq \theta(f g) \leq \theta(f)$, It follows that $0 \leq \theta_{g} \leq \theta$. Thus $\theta_{g}=\lambda_{g} \theta$ for a scalar $\lambda_{g}$ which can be easily evaluated to be $\theta(g)$, so that $\theta(f g)=\theta(f) \theta(g)$. This argument can be extended by standard means to show that $\theta$ is an algebra homomorphism on $I(1)$. We next consider nonnegative elements 8 In $I(1)$ and $f$ in $V$. To facilitate computations if $\theta(g)=0$, we let $g^{*}=g+1$. Then

$$
\begin{aligned}
\theta\left(f g^{*}\right) & =\bigvee_{n}\left[\theta\left(f g^{*}\right) \wedge n \theta\left(g^{*}\right)\right] \\
& =\bigvee_{n}\left[\theta(f \wedge n 1) g^{*}\right] \\
& =\bigvee_{n}\left[\theta(f \wedge n 1) \theta\left(g^{*}\right)\right] \\
& =\left[\bigvee_{n}(\theta(f) \wedge n)\right] \theta\left(g^{*}\right)=\theta(f) \theta\left(g^{*}\right),
\end{aligned}
$$

the third step being valid because $f \wedge \mathrm{nl}$ and $\mathrm{g}^{*}$ are in $I(1)$. Thus $\theta(f g)=\theta(f) \theta(g)$. The argument can now be repeated without the restriction 
that $g$ is in $I(1)$. The standard extension establishes that $\theta$ is an algebra homomorphism on $\nabla$.

EXAMPLE 1. The $\phi$-algebra $\mathcal{L}$ of all real-valued measurable functions on $[0,1]$ Is countably universally complete (hence, 2-universally complete) and is complete in the uniform topology. It is known (see [7]) that $\mathcal{L}$ is not isomorphic as a $\phi$-algebra to any function space $c(S)$. It follows from Lemma 2 that $\mathcal{L}$ is not isomorphic as a lattice to any $c(S)$. Thus $\mathcal{L}$ is not a uniformly dense sublattice of any $C(S)$. Furthermore, if $\mathscr{L}$ separated the points in $\beta X$ then the space of bounded functions in $\mathcal{L}$ would be $C(B X)$ by the Stone-Weierstrass theorem. But since $\mathcal{L}$ as a $\phi$-algebra is closed under inversion, this would imply $\mathscr{L}=c(X)$. Thus $\widetilde{\beta X}+\beta X$. To show $\mathscr{L}=\mathrm{K}(\mathrm{X}, \widetilde{\beta X})$, consider $f$ in $\mathrm{K}(\mathrm{X}, \widetilde{\beta X})$. Then $f$ is in $C\left(\widetilde{B X} \backslash \Lambda_{h}\right)$ for some $h$ in $F(X, \widetilde{B X})$. Since $\widetilde{B X} \backslash \Lambda_{h}$ is $\sigma$-compact, the topology of $C_{c o}\left(\widetilde{B X}-\Lambda_{h}\right)$ is metrizable. By the Stone-Weierstrass theorem there exists a sequence of functions in $\mathcal{L}$ convergent to $f$ in $C_{c o}\left(\tilde{\beta X} \backslash \Lambda_{h}\right)$. Thus $f$ on $[0,1]$ is a pointwise limit of a sequence of measurable functions, and so in $\mathscr{L}$. We remark that $x$ here is $[0,1]$ in the discrete topology and $k(X, \widetilde{B X})$ consists of just those continuous extended realvalued functions on $\widetilde{\beta} \widetilde{X}$ which are finite on a dense subset.

LEMMA 3. Let $V$ be 2-universally complete. For each function $g$ in $K(x, \widetilde{B X})$ there is a function $f$ in $V$ such that $g \leq f$.

PROOF. We begin by showing that for compact sets $K_{1}$ and $K_{2}$ in $\widetilde{\beta X}$ there is a function $v$ in $v$ which is zero on $K_{1}$, one on $K_{2}$ and satisfies $0 \leq v \leq 1$. Given $p$ in $K_{1}$ and $x$ in $K_{2}$, since $v$ is a vector space and separates $\widetilde{B X}$ there is a function $v_{x}$ in $v$ such that

$\begin{array}{ll}0 & \leq v_{\mathbf{x}}(p)<1<v_{x}(x) \\ \text { Clearly, } & 0 \leq v_{x}(q)<1<v_{x}(y)\end{array}$

for all points $q$ in some neighborhood $U_{x}$ of $p$ and all points $y$ in some neighborhood $N_{x}$ of $x$. Let $v_{p}$ be the supremum of functions $v_{x}$ corresponding to a finite subcover $\left\{\mathrm{N}_{x}\right\}$ of $\mathrm{K}_{2}$. Then

$$
0 \leq v_{p}(q)<1<v_{p}(y)
$$


for all $y$ in $K_{2}$ and $q$ in a neighborhood of $W_{p}$ of $p$. Letting $w$ be the infimum of functions $v_{p}$ corresponding to a finite subcover $\left\{w_{p}\right\}$ of $K_{1}$ we obtain

$$
0 \leq w(q)<1<\alpha<w(y)
$$

for all $q$ in $K_{1}, y$ in $K_{2}$ and some real number $\alpha$. The function

$$
v=\left\{\frac{1}{\alpha-1}[(w-1) v 0]\right\}^{\wedge} 1
$$

has the desired properties. Now let $\mathrm{g}$ be a function in $\mathrm{K}(\mathrm{X}, \widetilde{\beta X})$. By Lemma 1 there is a function $h$ in $F(X, \widetilde{B X})$ such that $h \geq g$. Consider the compact subsets

$$
A_{n}=h^{-1}[2 n-2,2 n]
$$

and

$$
\mathrm{B}_{\mathrm{n}}=\mathrm{h}^{-1}[0,2 \mathrm{n}-3] \cup \mathrm{h}^{-1}[2 \mathrm{n}+1, \infty]
$$

of $\beta X(n=1,2, \ldots)$ with the understanding that $B_{1}=h^{-1}[3, \infty]$. It follows from the first part of this proof that there is a function $v_{n}$ in $v$ with $0 \leq v_{n} \leq 2 n$ which has value $2 n$ on $A_{n}$ and is zero on $B_{n}$. Since $\left\{v_{n}\right\}$ is a 2-disjoint collection, its supremum is a function in $V$ greater than or equal to h (and hence g).

Given $u$ in $v^{+}$, we consider the ideal

$$
[\mathrm{u}]^{\mathrm{V}}=\{\mathrm{v} \in \mathrm{V}:|\mathrm{v}| \leq \lambda \mathrm{u} \text { for some } \lambda \text { in } R\}
$$

and we set, for each $v$ in $[u]$,

$$
|| v||_{u}=\inf \{\lambda>0:|v| \leq \lambda u\}
$$

It is easy to verify that the normed spaces

$$
\left\{\left([\mathrm{u}]^{\mathrm{V}},\|\cdot\|_{\mathrm{u}}\right): \mathrm{u} \in \mathrm{v}^{+}\right\}
$$

form an inductive system ordered by inclusion, whose locally convex inductive limit is $\mathrm{V}_{\mathrm{T}_{\mathrm{o}}}$ (see, e.g., $[13, \mathrm{p} .122]$ ).

PROPOSITION 4. Let $B$ be a 2-universally complete function lattice with 1 and having carrier space $X$. Then

$$
\mathrm{V}_{\mathrm{T}_{0}}=\mathrm{v} \cap \mathrm{K}_{\mathrm{T}_{0}}(\mathrm{x}, \widetilde{\beta \mathrm{X}})
$$

PROOF. We recall that $\mathrm{V}_{\mathrm{T}}$ is the locally convex inductive limit of the factors $\left\{\left([\mathrm{u}]^{\mathrm{V}},\|\cdot\| \|_{\mathrm{u}}\right): \mathrm{u} \in \mathrm{v}^{+}\right\}$, where $[\mathrm{u}]^{\mathrm{V}}$ is the ideal in $\mathrm{V}$ generated by u. It follows from Lemma 3 that $\mathrm{K}_{\mathrm{T}}(\mathrm{X}, \widetilde{\beta X})$ is the locally convex inductive limit of the factors $\left\{\left([u]^{K},\|\cdot\|_{u}\right): u \in v^{+}\right\}$, where $[u]^{K}$ is the 
ideal in $K(X, \widetilde{\beta X})$ generated by $u$. It is easy to verify that the topology of $\mathrm{V}_{\mathrm{T}_{0}}$ is finer than that of $\mathrm{V} \cap \mathrm{K}_{\mathrm{T}_{0}}(\mathrm{X}, \widetilde{\beta X})$. For the converse, let $U$ be a solid neighborhood of zero in $V_{T_{0}}$. Then for some collection $\left\{\alpha_{u}: u \in V\right\}$ of positive scalars, $U$ contains the convex hull of $U\left\{\alpha_{u}[-u, u]^{V}: u \in V\right\}$, where $[-u, u]^{V}$ denotes an order interval in $v$. Denoting by $[-u, u]^{\mathrm{K}}$ the order interval in $\mathrm{K}(\mathrm{X}, \widetilde{\beta X})$, we let $\mathrm{v}$ be in the intersection with $\mathrm{V}$ of the convex hull of $\cup\left\{\alpha_{u}[-u, u]^{K}: u \in v\right\}$. Since $v==_{i=1}^{n} \sum_{i} f_{i}$ for scalars $\lambda_{i}$ satisfying $\sum_{i=1}^{n}\left|\lambda_{i}\right| \leq 1$ and $f_{i}$ in $\alpha_{u_{i}}\left[-u_{i}, u_{i}\right]^{K}$, then

By the solidness of $u, v\left|\leq \sum_{i=1}^{n}\right| \lambda_{i}|\cdot| \alpha_{u_{i}} u_{i} \mid \in U$.

The next theorem is a consequence of Proposition 4 and Theorem 1 .

THEOREM 2. Let $\mathrm{V}$ be a 2-universally complete function lattice with 1. The order topology $T_{0}$ on $V$ is the topology of compact convergence on the carrier space of $\mathrm{V}$.

\section{CONVERGENCE STRUCTURES RELATED TO UNIFORM CONVERGENCE}

In this section we will be using the ideas of convergence space theory (see, e.g., $[1])$. We will consider convergence structures on $\mathrm{K}(\mathrm{X}, \mathrm{Y})$, where $\mathrm{Y}$ is compact and Hausdorff and $\mathrm{X}$ is realcompact in $\mathrm{Y}$. We let $\mathrm{K}_{\sigma}(\mathrm{X}, \mathrm{Y})$ denote the convergence space inductive limit of the system

$$
\left\{C_{c o}\left(Y \backslash \Lambda_{f}\right): f \in F(X, Y)\right\} \text {, }
$$

together with the continuous inclusion maps. Thus a net $\left\{g_{\alpha}\right\}$ converges to $g$ in $K_{\sigma}(X, Y)$ if and only if $g_{\alpha}$ is in a factor $C\left(Y \backslash \Lambda_{f}\right)$ for all $\alpha$ beyond some $\alpha_{0}$ and $\left\{g_{\alpha}\right\}_{\alpha} \geq \alpha_{0}$ converges to $g$ in $C_{c o}\left(Y \backslash \Lambda_{f}\right)$; equivalently, a filter $\theta$ converges to $g$ in $K_{\sigma}(X, Y)$ if and only if $\theta$ contains the neighborhood filter at $g$ in some factor $C_{c o}\left(Y \backslash \Lambda_{f}\right)$. We note that for $X$ realcompact, $K_{\sigma}(X, B X)$ is the convergence space $C_{I^{\prime}}(X)$ studied in [2].

$A$ set $A$ in a vector lattice $W$ is bounded if there is an element $w$ in $W$ such that $|a| \leq w$ for $a 11$ a in $A$. A filter $\Theta$ is bounded if some set $A \in \theta$ is bounded. It is easy to verify that if $W_{\delta}$ is a convergence vector lattice then the space $W_{\delta b}$ containing only the bounded filters from $W_{\delta}$ is 
also a convergence vector lattice. Thus $K_{\sigma b}(X, Y)$ is a convergence vector lattice. (A net $\left\{f_{\alpha}\right\}$ converges to a function $f$ in $K_{\sigma b}(X, Y)$ if and only if it converges in $K_{\sigma}(X, Y)$ and is bounded - i.e., there exists a function $g$ in $K(X, Y)$ and an index $\alpha_{0}$ such that $\left|f_{\alpha}\right| \leq g$ for all $\alpha \geq \alpha_{0}$ )

We note that relative uniform convergence on a vector lattice is a convergence vector lattice structure.

THEOREM 3. Let $\mathrm{V}$ be a function lattice with 1 . If $\mathrm{V}$ is 2-universally complete, the identity map from $v_{\rho}$ onto its image in $\mathrm{K}_{\sigma \mathrm{b}}(\mathrm{X}, \widetilde{\beta X})$ is bicontinuous. PROOF. Let net $\left\{v_{\alpha}\right\}$ converge to zero in $v_{\rho}$ : For some $u$ in $v$ and $\mathrm{n}=1,2, \ldots,\left|\mathrm{v}_{\alpha}\right| \leq \frac{1}{\mathrm{n}} \mathrm{u}$ for $\alpha \geq \alpha_{\mathrm{n}}$.

Thus $\left\{v_{\alpha}\right\}$ converges to zero in $C_{\rho}\left(\tilde{\beta X} \backslash \Lambda_{u}\right)$, where $x$ is the carrier space of $v$. It follows that $\left\{v_{\alpha}\right\}$ converges to zero in $c_{c o}\left(\widetilde{\beta X}-\Lambda_{u}\right)$ and hence in $\mathrm{K}_{\sigma \mathrm{b}}(\mathrm{X}, \widetilde{\beta X})$. Conversely, let net $\left\{\mathrm{v}_{\alpha}\right\}$ in $\mathrm{v}$ converge to zero in $\mathrm{K}_{\sigma \mathrm{b}}(\mathrm{X}, \widetilde{\beta X})$.

For some $g$ in $K(X, \widetilde{B X})$ and $\alpha_{0}$.

$$
\left|v_{\alpha}\right| \leq \mathrm{g} \text { for } \alpha \geq \alpha_{0} \text {. }
$$

By Lemma 3 we can assume that $g$ is in $v$; we can also assume $g \geq 1$ and $\left\{v_{\alpha}\right\}$ converges to zero in $C_{c o}\left(\tilde{B X} \backslash \Lambda_{g}\right)$. Thus, given $n$, there is an $\alpha_{n}$ such that for $x$ in $g^{-1}[1, n]$ $\left|v_{\alpha}(x)\right| \leq \frac{1}{n} \leq \frac{1}{n} g^{2}(x)$ for $\alpha \geq \alpha_{n}$.

For $x$ not in $g^{-1}[1, n]$, since $g^{2}(x)>n g(x)$,

$$
\left|v_{\alpha}(x)\right| \leq g(x)<\frac{1}{n} g^{2}(x) \text { for } \alpha \geq \alpha_{0} \text {. }
$$

By Lemma 3 there is a $w$ in $V$ such that $w \geq g^{2}$. Thus for $\alpha$ beyond $\alpha_{0}$ and $\alpha_{n}$,

$$
\left|v_{\alpha}\right| \leq \frac{1}{n} g^{2} \leq \frac{1}{n} \text { w. }
$$

We conclude that $\left\{v_{\alpha}\right\}$ converges to zero in $v_{\rho}$.

COROLLARY 1. For realcompact $X$,

$$
C_{\rho}(X)=K_{\sigma b}(X, \beta X)=C_{I^{\prime} b}(X) .
$$

We recall that a set $A$ in a convergence vector lattice $W_{\delta}$ is dense in $W$ If every element of $W$ is the limit in $\delta$ of a net in $A$. The space $W_{\delta}$ is complete if every Cauchy net (filter) converges. If $W_{\delta}$ is complete, it 
follows readily that $W_{\delta b}$ is complete.

THEOREM 4. Let $V$ be a 2-unfversally complete function lattice with 1 having carrier space $X$. Then $K_{\sigma b}(X, \widetilde{B X})$ is complete and contains $V$ as a dense subspace. Moreover, $V_{\rho}$ is complete if and only if it equals $k_{\sigma b}(\mathrm{X}, \widetilde{\beta X})$.

PROOF. The space $K_{\sigma}(X, \widetilde{B X})$, being an inductive limit of complete factors $C_{c o}\left(\widetilde{B X} \backslash \Lambda_{f}\right)$, is easily seen to be complete; thus $K_{\sigma b}(X, \widetilde{B X})$ is complete. Given $f$ in $K(X, \widetilde{B X})$, $f$ is in $C\left(\widetilde{\beta X} \backslash \Lambda_{g}\right)$ for some $g$ in $F(X, \widetilde{\beta X})$. Since $v \cap \mathrm{C}\left(\tilde{\beta X} \backslash \Lambda_{g}\right)$ is a sublattice of $\mathrm{C}\left(\tilde{\beta X} \backslash \Lambda_{g}\right)$ containing the constant functions and separating the points of $\tilde{B X} \backslash \Lambda_{g}$, there is a net $\left\{v_{\alpha}\right\}$ in $v$ converging to $f$ In $C_{c o}\left(\widetilde{B X} \backslash \Lambda_{g}\right)$ by the Stone-Welerstrass Theorem. By Lemma 3 there is a $w$ In $v$ with $w \geq|f| ;\left\{\left(v_{\alpha}{ }^{\wedge}\right) v(-w)\right\}$ converges to $f$ in $K_{\sigma b}(x, \widetilde{B X})$. The last statement of the theorem is now a consequence of Theorem 3 .

It follows from Theorem 4 that if a 2-universally complete function lattice $V$ with 1 separates $B X$ and if $v_{\rho}$ is complete, then $V=C(X)$. If, moreover, $X$ is $\sigma$-compact and locally compact then $K_{\alpha}(X, B X)=C_{c o}(X)$ since $B X \backslash X=\Lambda_{f}$ for some $f$ in $F(X, B X)=C(X)$, implying $v_{\rho}=C_{c o b}(X)$.

COROLLARY 2. The space $\mathcal{L}_{p}$ of all real-valued measurable functions on $[0,1]$ with the relative uniform convergence structure is complete.

PROOF. It was shown in Example 1 that $\mathcal{L}=\mathrm{K}(\mathrm{X}, \widetilde{\beta} \widetilde{\mathrm{X}})$.

We cite two examples to show that "relatively uniformly complete" and "2-universally complete" are independent concepts.

EXAMPLE 2. Let $V$ be the space of continuous functions $f$ on the real line such that the restriction of $f$ to any compact set consists of finitely many line segments. Clearly, $V$ is a function lattice containing 1 . To see that $V$ is 2-umiversally complete, let $G$ be a 2-disjoint collection of functions in $V$ and let $K$ be a compact subset of $R$. For each $y$ in $K$ there is a function $f_{y}$ in $G$ such that $\left|f_{y}\right|(y)>0$, since $y$ is in the carrier space $x$ of $v$. Thus, $\left|f_{y}\right|(z)>0$ for all $z$ in some neighborhood of $y$. Since finitely many such neighborhoods cover $K$ there are finitely many functions $\left|f_{y}\right|$ whose supremum $f$ is positive on $K$. It follows that $|g| \wedge f \neq 0$ for at most 
finitely many $g$ in $G$; 1.e., $g(K)=0$ for all but finitely many $g$ in $G$. Thus, since on any compact set the pointwise supremum of $G$ is a supremum of finitely many functions, $G$ has a supremum in V. However, any continuous function which vanishes outside the interval $(0,1)$ is a relative uniform limit of functions in $\nabla$; thus $V$ is not relatively unfformly complete.

EXAMPLE 3. We will call a function on the reals $R$ "ultimately a polynomial" if it is continuous and is equal to a polynomial on the complement of some Interval $[-n, n]$ and we let $V$ be the solid hull in $C(R)$ of the set of functions which are ultimately polynomials. We will argue that the carrier space $\mathrm{X}$ of $\mathrm{V}$ is $R$. Where $C^{\circ}(R)$ is the space of bounded continuous functions on $R$,

$$
\mathrm{C}^{\circ}(R) \subseteq \mathrm{C} \subseteq \mathrm{C}(R)
$$

Since $V$ separates $R$ and $V \cap C^{\circ}(R)$ separates $X$, we can assume (examining the adjoint maps)

$$
\text { BR } 2 x \supseteq \mathbf{R} .
$$

Letting $f$ denote the extended real-valued function on $B R$ whose restriction to $R$ is $f(x)=x$, we will prove that $f$ is infinite on $B R \backslash R$. If $s 0$, then $x=\mathbb{R}$ since $f$ must be finite on $x$. Let $p$ be a point in $B R \backslash R$ and $\left\{r_{\alpha}\right\}$ a net In $R$ convergent to $p$. Then $\left\{f\left(r_{\alpha}\right)\right\}$ converges to $f(p)$. If $f(p)$ were real, we would conclude that $f(p)=p$ by uniqueness of the limit of $\left\{r_{\alpha}\right\}$ in $B R$, a contradiction. We can now show that $V$ is not 2-universally complete. Let $\left\{f_{n}\right\}_{n=-\infty}^{\infty}$ be a collection of continuous functions on $R$ chosen so that $f_{n}(x)$ is zero outside the interval $(2 n-3,2 n+1)$ and equal to $e^{x}$ on the interval $[2 n-2,2 n]$. Clearly, $\left\{f_{n}\right\}$ is a 2-disjoint collection in $V$ with no supremum in $v$. On the other hand, if $\left\{v_{\alpha}\right\}$ is a relatively uniformly Cauchy net in $v$, there Is a strictly positive function $w$ in $v$ such that for $\alpha, \beta \geq \gamma_{n} \quad(n=1,2, \ldots)$

$$
\left|v_{\alpha}-v_{\beta}\right| \leq \frac{1}{n} w .
$$

Clearly $\left\{v_{\alpha}\right\}$ is bounded in $v$, and since $w$ is bounded on each compact set, $\left\{v_{\alpha}\right\}$ is Cauchy in $C_{c o}(R)$. Thus $\left\{v_{\alpha}\right\}$ converges in $C_{c o}(\mathbb{R})$ to some function $f$ in $C(R)$. It follows that for all $\alpha \geq \gamma_{n}$

$$
\left|v_{\alpha}-f\right| \leq \frac{1}{n} w
$$


Thus $|f|$ is bounded by the function $\left|v_{\gamma_{1}}\right|+w$ (which is in $v$ ) so that $f$ is in $v$, and $\left\{v_{\alpha}\right\}$ converges relatively uniformly to $f$ in $v$. Hence, $v$ is relatively uniformly complete.

\section{$\underline{\text { References }}$}

1. E. Binz, Continuous Convergence on $C(X)$, Lecture Notes in Mathematics 469 (Springer-Verlag, Berlin 1979).

2. E. Binz and W. Feldman, On a Marinescu structure on $C(X)$, Comment. Math. Helv. 46 (1971), 436-450.

3. W. A. Feldman and J. F. Porter, A vector lattice topology and function space representation, Trans. Amer. Math. Soc. 235 (1978), 193-204.

4. L. Gillman and M. Jerison, Rings of Continuous Functions, (D. Van Nostrand, Princeton 1960).

5. H. Gordon, Relative uniform convergence, Math. Ann. 153 (1964) 418-427.

6. E. Hewitt, Linear functionals on spaces of continuous functions, Fund. Math. 37 (1950), 161-189.

7. M. Henriksen and D. G. Johnson, On the structure of a class of archimedean lattice-ordered algebras, Fund. Math. 50 (1961), 73-94.

8. D. Hsieh, Topological properties associated with the $\lambda$-map, Bull. Acad, Polon. Sct. Ser. Sci. Math. Asronom. Phys. 20 (1972), 1-6.

9. K. Kutzler, Eine Charakterisierung von Lindelöf-Räumen, Arch. der Math. 26 (1975), 214-221.

10. E. R. Lorch, Compactification, Baire functions, and Daniel integration, Acta Sci. Math. 24 (1963), 204-218.

11. L. Nachbin, Topological vector spaces of continuous functions, Proc. N.A.S. 40 (1954), 471-474.

12. A.Peressint, Ordered Topological Vector Spaces, (Harper and Row, NY 1967).

13. H. Schaefer, Banach Lattices of Positive Operators, (Springer-Verlag, Berlin 1974). 


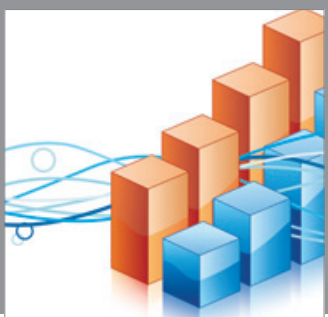

Advances in

Operations Research

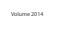

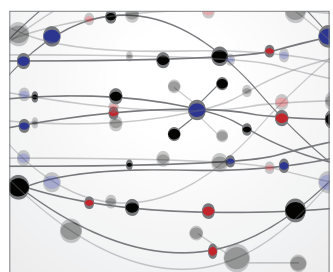

\section{The Scientific} World Journal
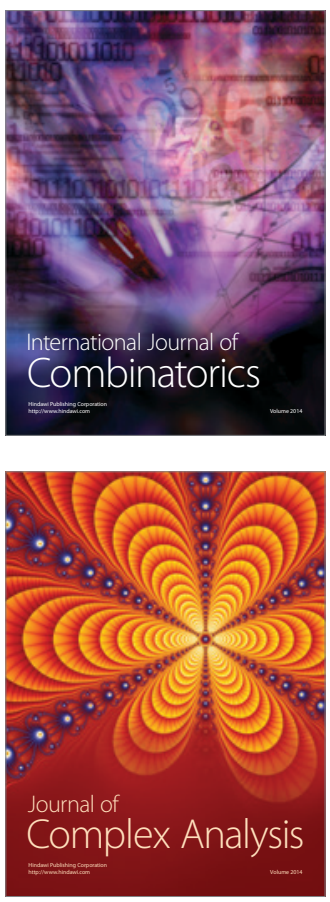

International Journal of

Mathematics and

Mathematical

Sciences
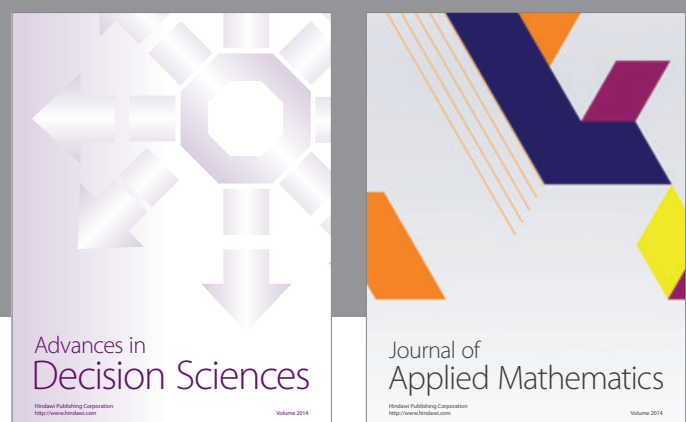

Journal of

Applied Mathematics
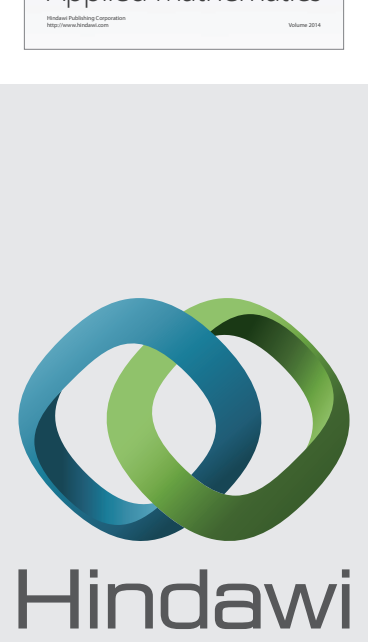

Submit your manuscripts at http://www.hindawi.com
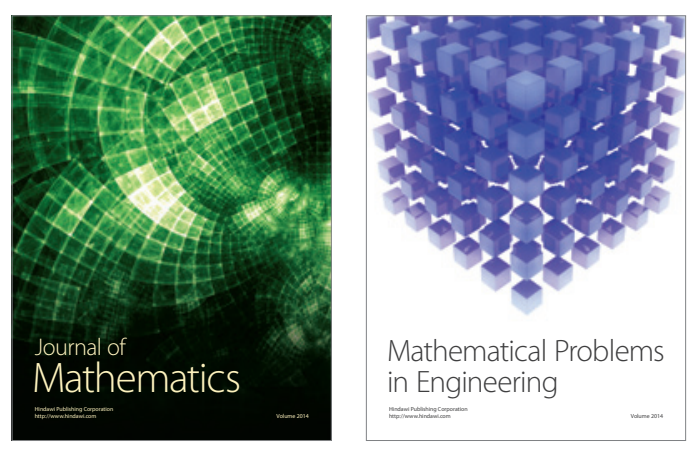

Mathematical Problems in Engineering
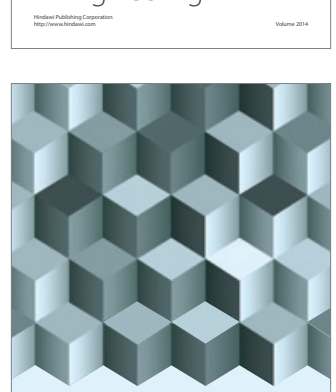

Journal of

Function Spaces
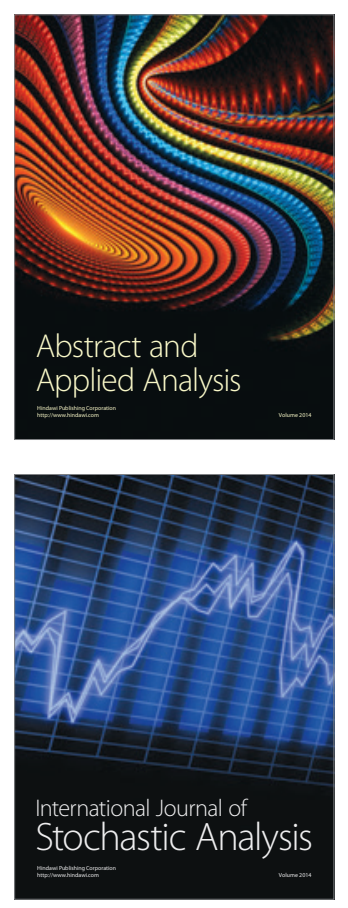

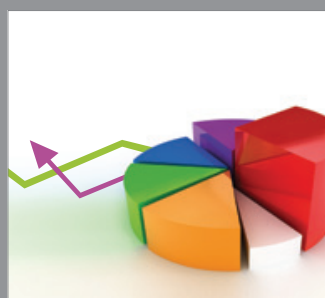

ournal of

Probability and Statistics

Promensencen
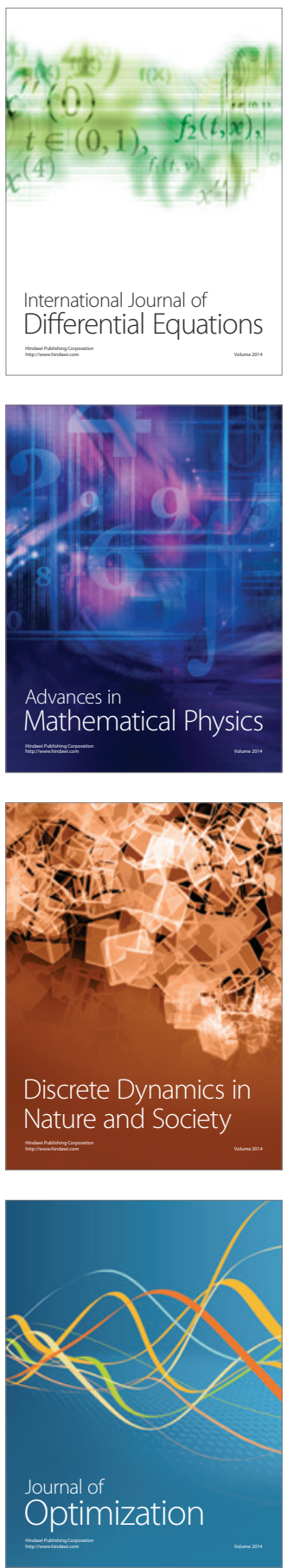